\title{
ENFERMEDAD DE CHAGAS EN EL SUR DEL PERU
}

\section{Estudio epidemiológico, clínico, electrocardiográfico y profiláctico*}

\author{
Alberto Cornejo-D. - Edgardo Cubas-N. - Guillermo Eyzaguirre \\ Pedro Domínguez - Herbert Bitrich - Rolando Gómez-C. \\ julia Cornejo-s.
}

Desde 1919, (1) se sabe de la presencia de casos de enfermedad de Chagas en el Perú y dos años antes, en 1917 (2), se demostró la presericia de triatóminos infectados, en condiciones naturales, por $T$. cruzi en un Departamento del Sur de la República. A pesar de los 45 años transcurridos, no se tiene idea precisa sobre todos los aspectos epidemiológicos de esta enfermedad, tampoco se conoce, con precisión, la frecuencia con que se presentan síntomas y signos dependientes de compromiso cardíaco y, finalmente, en muy pocas oportunidades, se han hecho investigaciones de orden profiláctico, relacionadas con el insecto transmisor.

En la amplia revisión bibliográfica que tuvimos oportunidad de efectuar (3) en 1958, destaca la acentuada orientación del esfuerzo de los investigadores peruanos hacia el conocimiento de algunos aspectos epidemiológicos de la enfermedad, especialmente, los relacionados con los triatóminos, su distribución, hábitos y rol transmisor. También se han estudiado las características farvorables del tipo de vivienda en

- Investigaciones realizadas en la Facultad de Medicina de San Fernando, con fondos de la partida para la investigación científica y presentado a 10s Séptimos Congresos Internacionales de Medicina Tropical y Paludismo. Río de Janeiro, 1963. 
las zonas endémicas, la presencia de reservorios y, en mucho menor escala, se ha tratado de determinar la incidencia en distintas zonas del territorio peruano.

Era interesante, a fin de completar nuestros estudios, realizar un esfuerzo, para obtener información referente a la frecuencia con que se presentan manifestaciones cardíacas en los enfermos de Tryponosomiasis Americana en zonas donde las condiciones epidemiológicas habían demostrado que esta enfermedad es endémica.

Tal estudio se hacía necesario en el Perú ya que investigaciones similares efectuadas en otros países de América, habían demostrado resultados diferentes en lo que a compromiso cardíaco se refiere (A), (5), (6), (7), (8).

También hemos considerado interesonte realizar investigaciones de orden profiláctico, relacionadas con la efectividad del insecticida GAMMA BHC CERECLOR sobre el Triatoma infestans, único transmisor en la región sud-occidental del Perú.

En esta oportunidad damos a conocer los resultados de un estudio epidemiológico, clínico, electrocardiográfico y profiláctico, efectuado en dos Departamentos del Sur de nuestro país, donde previamente se había comprobado la presencia de todos los factores epidemiológicos necesarios para que fuesen considerados como zonas endémicas de enfermedad de Chagas.

El primer caso de cardiopatía chagásica conocido en el Perú pertenece al Dr. R. Alzamora F. (9), quien estudió un paciente en 1928 y recién en 1958 dio a publicidad su hallazgo clínico, que lamentablemente no pudo ser confirmado con pruebas diagnósticas. Se trató de un enfermo con miocarditis crónica muy avanzada, cardiomegalia, sin lesión valvular ni hipertensión y marcada insuficiencia cardíaca, tenía un corazón con diámetro transversal $156 \mathrm{~mm}$. y que en los electrocardiogramas presentó fibrilación auricular, aleteo auricular, bloqueo de rama derecha intermitente, momentos de paro cardíaco, bloqueo sino-auricular y complejos nodales pareados. Este es un caso indudable de cardiopatía chagósica en opinión de los cardiólogos y especialistas en enfermedad de Chagas más destacados de América. Posteriormente, A. Peralta, Cornejo-Donayre y col. informaron el diagnóstico de un paciente con cardiopatía chagásica inicial (10) y el caso de una madre multípara de 40 años que presentó eclampsia y edema agudo pulmonar dependiente de cardiopatía chagásica confirmada por el electrocardiograma, xenodiagnóstico y reacción de fijación del complemento (11). 
A. Peralta (12), Montesinos y col. (13), refieren el estudio clínico y electrocardiográfico de 14 sujetos de 4 a 19 cños con xenodiagnóstico positivo y que no presentaban sintomatología destacada atribuíble a cardiopatía. Los electrocardiogramas de todos ellos, correspondíon $a$ trazados normales en relación con su edad y sólo destacon tres, con caracteres que podrían interpretarse en relación con cardiopatía "potencial" ya que tenían xenodiagnóstico positivo. Uno era niño, de 4 años que tenía PR 0.17" y frecuencia cardíaca de 115 por minuto, lo que, según las tablas de Ashman, es ligeramente oumentado y sugeriría bloqueo incompleto aurículo-ventricular. En dos casos de este mismo grupo, ambos de 13 años, se encontró en V-1, RS equipotencial con onda $T$ negativa, lo que podría ser normal en niños o corresponder a bloaueo incompleto de rama derecha.

Los Departamentos del Sur del Perú escogidos para efectuar estas investigarciones fueron Arequipa y Moquegua, en los cuales se seleccionaron los valles de Majes y Moquegua respectivamente, por ser zonas donde numerosos estudios habían demostrado de manera irrefutable, la presencia de factores epidemiológicos que las hacían zonas endémicas de Trypanosomiasis Americana (14) (15) (16) (17) (18).

El valle de Majes está situado en la provincia de Castilla del Departamento de Arequipa, se extiende desde los $16^{\circ}$ hosta los 16.18' de latitud sur y su ancho estớ comprendido entre los $72^{\circ} 25^{\prime}$ y $72^{\circ} 36^{\prime}$ de longitud oeste del meridiano de Grenwich. Su altura varía entre los 400 y 700 metros sobre el nivel del mar y su temperatura durante el año, fluctúa entre los 18 y $30^{\circ} \mathrm{C}$., correspondiendo las cifras más elevadas a los meses de Enero, Febrero y Marzo.

El valle de Moquegua está situado a los 17:11'39” de latitud sur y 70 ${ }^{\circ} 56^{\prime} 04^{\prime \prime}$ de longitud oeste del meridiano de Grenwich. Este valle se encuentra entre los 1400 y 1500 metros de altura sobre el nivel del mar, su clima es sub-tropical y caluroso durante los doce meses del año.

\section{MATERIAL Y METODOS}

Las investigaciones en el valle de Majes se efectuaron en el año 1958 e incluyeron a 461 residentes de la zona, que no habían sido previamente seleccionados. De éstos, sólo consideramos 259 en la presente comunicación, debido a que en ellos fue poslble realizar por lo menos dos de las pruebas y exámenes utilizados con fines diagnósticos y de exploración cardiológica, es decir, electrocardiograma, reacción 
de Guerreiro-Machado y xenodiagnóstico. En muchos de los restantes se perdieron los electrocardiogramas durante el revelado y en otros, no fue posible obtener muestras de songre o no se practicó la prueba de! xenodiagnóstico.

Entre los 259 hubo 120 hombres y 139 mujeres, todos habian nacidc o residido durante tiempo prolongado en el valle de Majes y el de mayor edad tenía 90 oños y el más joven 3 años.

Los grupos por edad se clasificaron de la siguiente manera: menores de 9 oños, 15; de 10 a 19, 135; de 20 a 39 años, 57; de 40 a 59 años, 39; y de 60 y más oños, 13.

Las investigaciones en el valle de Moquegua, han sido realizadas a partir de Marzo de 1963 y en ellas se incluyen 512 personas de la población general y sin previa selección, de las que 404 habían nacido y tenían muy prolongada residencia en la localidod; de los restantes, 106 habión nacido en otros Departamentos del Perú y dos eran extrarıjeros; pero todos ellos, con excepción de 7, residían en Moquegua más de un año. Entre los 512 examinados, hubo 268 hombres y 244 mujeres. El más joven fue de 4 años y el de mayor edad tenía 90. Los grupos de edad fueron: menores de 9 años, 63; de 10 a 19 años, 134; de 20 a 39, 145; de 40 a 59 años, 129; y de 60 y más, 41.

Noventicuatro reacciones de Guerreiro-Machado correspondientes a igual número de residentes fueron realizados en el Departamento de Parasitología de la Universidad de Chile, por gentileza del Prof. A. Neghme y 459, correspondientes al grupo estudiado en Moquegua, se efectuaron en nuestro laborotorio.

En la prueba del xenodiagnóstico, se utilizó diversos estadios de $T$. intestans procedentes de nuestros criaderos y que habian sido alimentados exclusivamente con sangre de gallina.

En los electrocardiogramas practicados en el valle de Majes, se utilizó electrocardiógrałos a pilas marca "Sanborn" y un electrocardiógrafo fotográfico, mientras que para los electrocardiogramas practicados en Moquegua, se emplearon electrocardiógrafos de visión directa (en un 97\%) Samborn, modelo 51, Viso-Cardiette. La standarización fue de un milivoltio y en algunos se empleó medio milivoltio.

En las investigaciones de orden profiláctico, hemos usado GAMMA BHC CERECLOR fabricado por la Plant Protection Lmtda. de Londres, que fue puesto a nuestra disposición por los fabricantes. Este es un nuevo insecticida con $26 \%$ de isómero gamma tipo lindamo, que según los fabricantes tiene gran efectividad contra los triatórninos, ninguna acción tóxica para personas y animales y con acción residual de larga duración. 
En fichas clínico-epidemiológicas, previamente elaboradas, se anotaron los datos más importantes, remarcando, lugar de nacimiento, tiempo de residencia en la zona estudiada, características de la vivienda en relcción con el material de construcción y presencia de grietas y resque brajaduras favorables para criaderos de triatóminos; presencia de animales domésticos en la vivienda, no solamente aquellos que como las aves de corral sirven de fuente nutritiva de los triatomas, sino, especialmente, todos los que viven cerca del hombre y han probado servir de reservorio del Trypanosoma cruzi. En esta ficha se consignó tam. bién, los datos de la historia clínica completa, el examen físico general y especial, sobre todo del aparato cardio-vascular y de los resultados del electrocardiograma, del xenodiagnóstico y de la reacción de fijación del complemento.

En el examen electrocardiográfico, se han tomado las derivaciones standard y seís pre-cordiales, en cada uno de los sujetos considerados en la encuesta. Las tablas de Ashman han sido consideradas en la interpretación de los resultados.

Los xenodiagnósticos fueron sometidos a dos exámenes; el primero después de 15 días de su aplicación y el segundo, consistente en la disección, después de 60 días, examinando cuidadosamente el intestino medio y posterior.

La reacción de Guerreiro-Machado, que se practicó en los sujetos del valle de Majes, fue realizada en el Departamento de Parasitología de la Universidad de Chile y efectuada por la Dra. F. Knierim, siguiendo la técnica del 50\% de hemólisis de Bozicevich (19). En 459 sujetos del valle de Moquegua, practicamos la reacción de Guerreiro-Machado, utilizando antígeno preparado por nosotros a partir de cepas peruanas y siguiente la técnica utilizada en la Universidad de Chile (20) (21).

Se ha efectuado la búsqueda de triatóminos en 45 casas de los tres pobiados más importantes del valle de Majes, que representan poco más del $10 \%$ de viviendas de esa zona y, en todas ellas se capturó especímenes de Triatoma infestans en diversos estadios evolutivos.

En la ciudad de Moquegua y el caserío de Samegua, que son dos de los centros más importantes del valle, se efectuó la búsqueda y captura de triatóminos en 107 casas, que representan el 10\% de las viviendas de esos lugares. La captura se efectuó con ayuda de pinzas y linternas.

En el valle de Majes se capturó 294 especímenes de T. infestans y en el de Moquegua 697. Todos ellos fueron disectados, investigándose la presencia de T. cruzi. 
Se ha investigado la presencia de T. cruzi en la sangre circulante de animales domésticos encontrados en las viviendas de Moquegua. Practicamos 320 xenodiagnósticos en igual número de animales, entre los que hubo 113 perros, 68 gatos, 62 cuyes, 30 conejos, 27 cerdos, 8 cabalios, 6 burros y 6 cabras.

El rociado del insecticida GAMMA BHC CERECLOR, estuvo a cargo del Servicio Nacional de Erradicación de la Malaria y fue dirigido por la Entomóloga Srta. Carmen Villanueva, obteniéndose una concentración de 2 gr. por metro cuadrado y aplicando el rociado en el interior de toda la vivienda (paredes y techos), gallineros y corrales anexos a cada vivienda.

\section{RESULTADOS OBTENIDOS}

Tanto el valle de Majes como el de Moquegua, reúnen condiciones y características geográficas y climatéricas muy favorables para la presencia de $T$. infestans. El primero, tiene centros poblados cuya altura está entre 400 y $700 \mathrm{mt}$. sobre el nivel del mar y en el segundo lás ciudades más importantes se encuentran entre los 1400 y 1500 mt. En ambos lugares, la precipitación pluvial es escasa y el clima es caluroso duronte todo el año, correspondiendo a los meses de Diciembre, Enero, Febrero y Marzo los valores más elevados. La temperatura fluctúa entre 16 y $30^{\circ} \mathrm{C}$ durante el año. En Majes hay más humedad que en Moquegua. Esta última ciudad es el único centro poblado que tiene servicios de agua y desagüe.

La característica de lás viviendas en el valle de Majes es más o menos semejante en todos sus pueblos y caseríos. Predomina el tipo de construcción con material de adobe o paredes de "quincha" (cañas huecas), generalmente mal rebocadas con barro o yeso, observándose frecuentemente la presencia de numerosas resquebrajaduras y grietas que sirven de criaderos al $T$. intestans. Los techos son de caña revestida con barro.

En los caseríos más pequeños, las viviendas tienen paredes y techos de caña sin rebocar. Los pisos son de tierra en la gran mayoría y sólo en pocas, de cemento, ladrillo o madera.

En la cludad de Moquegua y en ei caserío de Samegua, las viviendas son muy amplias y están construídas preferentemente con adobe; los techos son altos, hechos con caña y de forma característica, en caballete, que da la apariencia de tratarse de casas de dos pisos; en muchas de ellas hay un cielo raso elaborado con "tocuyo" grueso y de 
jando amplio espacio, triangular, que fácilmente se convierte en criadero de "chirimachas".

Las casas tienen como promedio, tres habitaciones y además, disponen de sitios adyacentes para corrales, gallineros y chiqueros. Todos estos lugares albergan animales domésticos (cuyes, conejos, perros, gatos, cerdos y carneros), que como fuente de alimentación de fácil acceso para los triatóminos se convierten en magníficos criaderos, desde donde fácilmente llegan a los dormitorios. La vivienda está sobrepoblada, el hacinamiento destaca con caracteres alarmantes en el valle de Majes donde se ha encontrado un promedio de dos personas por coma.

En la totalidad de viviendas de las zonas estudiadas en los dos valles se creían omimales domésticos y en más del $50 \%$ de ellas se ha verificado la presencia de más de una especie.

Hemos practicado 320 xenodiagnósticos a igual número de animales domésticos, obteniéndose los resultados que a continuación se indican :hubo 12 positivos entre 113 perros; 11 positivos en 62 cuyes; 2 conejos positivos en 30 examinados y 2 positivos entre 68 gatos sometidos a este examen. Resultaron negativos el tolal de 27 cerdos, 8 caballos, 6 cuyes y 6 cabras. En el Cuadro $N^{9} 1$ se aprecian los resultados en amirrales de la ciudad de Moquegua y el caserío de Samegua.

\section{CUADRO N: 1}

RESULTADO DE 320 XENODIAGNOSTICOS PRACTICADOS EN ANIMALES DOMESTICOS DE MOQUEGUA Y SAMEGUA

\begin{tabular}{|c|c|c|c|c|c|c|c|c|c|c|c|c|c|c|c|c|c|}
\hline \multirow[b]{2}{*}{ Lugar } & \multicolumn{2}{|c|}{ Perros } & \multicolumn{2}{|c|}{ Cuyes } & \multicolumn{2}{|c|}{ Conejos } & \multicolumn{2}{|c|}{ Gatos } & \multicolumn{2}{|c|}{ Cerdos } & \multicolumn{4}{|c|}{ Caballos Burros } & \multicolumn{3}{|c|}{ Cabras } \\
\hline & E. & P. & E. & P. & E. & P. & E. & P. & E. & P. & E. & P. & E. & P. & E. & P. & TOTAL \\
\hline Somegua & 38 & 7 & 31 & 5 & 8 & 1 & 13 & 1 & 12 & 0 & 4 & 0 & 3 & 0 & 2 & 0 & 111 \\
\hline Moquegua & 75 & 5 & 31 & 6 & 22 & 1 & 55 & 1 & 15 & 0 & 4 & 0 & 3 & 0 & 4 & 0 & 209 \\
\hline TOTAL & 113 & 12 & 62 & 11 & 30 & 2 & 68 & 2 & 27 & 0 & 8 & 0 & 6 & 0 & 6 & 0 & 320 \\
\hline
\end{tabular}

E.- Número de examinados

P.- Número de positivos.

Durante los doce meses anteriores a la fecha que efectuamos los estudios, tomto en Majes como en Moquegua, no se había utilizado insecticida ni ofra protecición de la vivienda contra triatóminos.

En las 45 casas de Majes y 107 de Moquegua, que representan el $10 \%$ del total de las viviendas, se buscó triatóminos. Se capturó un 
total de 294 especímenes de T. infestans en diversos estadíos evolutivos en el $100 \%$ de las casas visitadas en Majes, los que estaban infectados por T. cruzi en el $51.02 \%$. El $80 \%$ de las casas visitadas en Majes teníar triatomas infectados.

En Moquegua se efectuó la búsqueda en 107 casas, logrando capturar diversos estadíos de esta especie en 101, lo que representa el 93.45\%. En estas viviendas se capturó en total 697 especímenes, de los que se examinó 681 correspondientes a 96 casas y encontróndose que el $17.32 \%$ estaban infectados con el trypanosoma.

De 259 personas examinadas en Majes, a 258 se practicó el xenodiagnóstico, resultando 7 positivos. Solamente en 94 de ellas fue posible realizar la reacción de Guerreiro-Machado que resultó positiva en 27; 4 fueron sospechosas y dos onticomplementarias. El total de positivos fue 32 , pues en dos se obtuvo positividad tanto en el xenodiagnóstico como en la reacción de fijación del complemento.

En Moquegua se examinaron 512 personas, de las cuales en 494 se practicó xenodiagnóstico, resultando 34 positivos. Del total de examinados fue posible practicar la reacción de Guerreiro-Machado sólo en 459, resultando 64 positivos, 19 sospechosos y 24 anticomplementarias. Al igual que en el grupo de Majes, en determinado número (25 casos), se obtuvo positividad en el xenodiagnóstico y en la reacción de fijación del complemento, por lo que el total de positivos lue de 73.

CUADRO N: 2

CASOS DE ENFERMEDAD DE CHAGAS DIAGNOSTICADOS ENTRE 259 RESIDENTES DE MAJES Y 512 DE MOQUEGUA

Método de diagnóstico empleado

\begin{tabular}{lccc}
\hline Lugar & Xenodiagnóstico & $\begin{array}{c}\text { Reacción de fijación } \\
\text { del complemento }\end{array}$ & Total \\
\hline Majes & 7 & 27 & 32 \\
Moquegua & 34 & 64 & 73 \\
Total & 41 & 91 & 105 \\
\hline
\end{tabular}

En algunos casos el diagnóstico se confirmó por ambos métodos. 
De los 259 sujelos del valle de Majes, sometidos al examen clínico general y especial de aparato cardiovascular, practicado por los cardiólogos doctores P. Domínguez y H. Bitrich, se encontró 67 con signos cardiológicos atribuíbles a compromiso cardíaco:

Ruidos cardíacos apagados ....................... 3

Primer ruido cardíaco intenso en punta .............. I

Segundo ruido cardíaco disminuído en foco aórtico ......... 1

Segundo ruido cardíaco aumentado de intensidad en foco

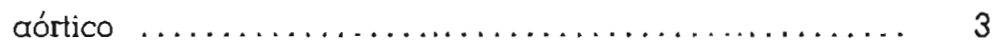

Primer ruido aumentado de intensidad en toco pulmonar .... 1

Segundo ruido aumentado de intensidad en foco pulmonar .. 2

Primer ruido desdoblado en punta ................... 8

Segundo ruido desdoblado en punta ................. 4

Primero y Segundo ruidos desdoblados en punta .......... 2

Primer ruido desdoblado en foco pulmonar ............... I

Segundo ruido desdoblado en foco pulmonar ............. 1

Arritmia sinusal ............................... 1

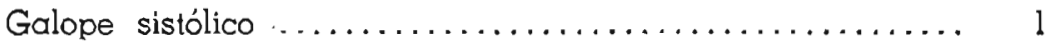

Soplo sistólico grado I - II/VI, en punta ............... 15

Soplo sistólico grado III - IV/VI en punta ............. 5

Soplo mesosistólico grado I - II/VI, en punta ............. I

Soplo sistólico grado I - II/VI, en foco aórtico ............ 4

Soplo sistólico grado III - IV/VI, en foco aórtico .......... 2

Soplo sistólico grado I - II/VI, en foco pulmonar .......... 17

Soplo sistólico grado III - IV/VI en foco pulmonar ........ 1

Soplo mesosistólico grado I-II/VI, en foco pulmonar ....... 2

El examen electrocardiográfico fue practicado en 243 sujetos de este grupo, encontrándose que en 206 era normal, confirmándose sólo en 37 las siguientes alteraciones electrocardiográficas:

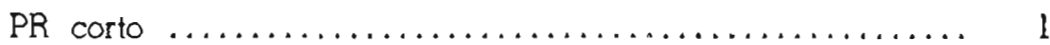

B. C. R. D. ................................ 5

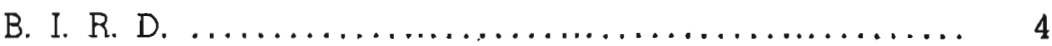

B. A. V. de primer grado ....................... 15

Onda $\mathrm{T}$ aplanada en precordiales izq. ................ 1

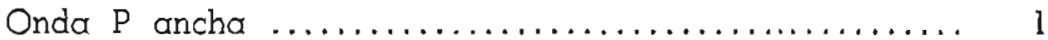

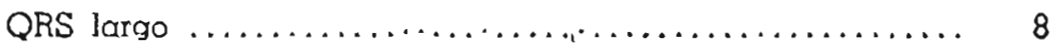

Eje eléctrico a la derecha ......................... 2

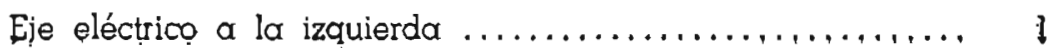


La distribución tanto de los E. C. G. normales como de los anormales según los grupos de edad es como sigue:

\section{CUADRO NO 3}

ELECTROCARDIOGRAMAS EN 243 RESIDENTES DEL VALLE DE MAJES SEGUN GRUPOS DE EDAD

\begin{tabular}{lcrr}
\hline $\begin{array}{c}\text { Grupos de } \\
\text { edad }\end{array}$ & $\begin{array}{c}\text { E. C. G. } \\
\text { efectuados }\end{array}$ & $\begin{array}{c}\text { E. C. G. } \\
\text { normales }\end{array}$ & $\begin{array}{c}\text { E. C. G. } \\
\text { anormales }\end{array}$ \\
\hline Menos de 9 años & 15 & 15 & 0 \\
$10-19$ & 125 & 111 & 14 \\
$20-39$ & 54 & 47 & 7 \\
$40-59$ & 35 & 27 & 8 \\
60 y más & 14 & 6 & 8 \\
Total & 243 & 206 & 37 \\
\hline
\end{tabular}

Se confirmó el diagnóstico de enfermedad de Chagas en 32 sujetos del grupo estudiado en Majes y entre ellos, solamente en 15 se obtuvo electrocardiogramas aprovechables de los cuales 13 estaban en límites normales y dos presentaban Bloqueo-A - V. de primer grado.

Los síntomas y signos enconirados en estos 32 casos fueron los siguientes:

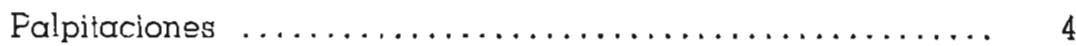

Disnea de esfuerzo $\ldots \ldots \ldots \ldots \ldots \ldots \ldots \ldots \ldots \ldots \ldots \ldots \ldots$

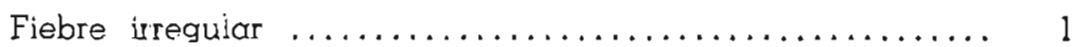

Primer ruido desdoblado en punta .................. I

Primero y segundo ruido desdoblado en punta ............ 1

Segundo ruido intenso en foco aórtico ................ 2

Segundo ruido desdoblado en foco pulmonar .............. 1

Soplo sistólico grado I - II/VI, en punta .............. 1

Soplo sistólico grado III - IV/VI, en punta ............. 2

Soplo mesosistólico grado I - II/VI, en foco pulmonar ...... 2

Soplo sistólico grado I - II/VI, en foco pulmonar .......... 3

Soplo sistólico grado I - II/VI, en foco aórtico ............ 1

No presentaron síntomas, sig̣noș ni altẹraciones E. C. G. ... 5 
No presentaron síntomas, signos y sí alteraciones E. C. G. . $\quad 0$

Presentaron síntomas, signos y alteraciones E. C. G. ....... 2

Presentaron síntomas, signos y no alteraciones E. C. G. ..... 8

Los resultados obtenidos en el examen clínico y electrocardiográfico de los 512 residentes del valle de Moquegua, revelaron que 160 presentaron alleraciones clínicas y/o electrocardiográficas que podrían estar relacionadas can cardiopatía.

En $€$ ste grupo se encontró los siguientes signos clínicos atribuíbles a compromiso cardíaco:

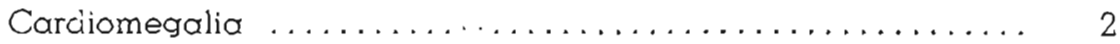

Səgundo ruido cumentado de intensidad en foco aórtico .... 3

Primer ruido disminuído de intensidad en foco pulmonar ... 1

Segundo ruido c.umentado de intensidad en foco pulmonar ... 3

Soplo sistólico grado I - II/VI, en punta ............... 5

Soplo sistólico grado II - IV/VI, en punta ............. 3

Soplo sistólico grado I - II/VI, en mesocardio ............ I

Soplo sistólico y diastólico grado III - IV/VI, en punta ..... I I

Soplo sistólico supraesternal grado I - II/VI ............ 3

Soplo sistólico grado I - II/VI, en foco aórtico ............ 2

Soplo sistólico grado III - IV/VI, en foco aórtico .......... 3

Soplo diastólico grado II/VI, en foco aórtico ............ I I

Soplo sistólico grado II/VI, en foco pulmonar ............ 1

Hipertensión arterial sistólica .................... 9

Hipertensión arterial sisto-diastólica ................. 2

Las alteraciones electrocardiográficas correspondientes a los referidos 160 sujetos fueron las siguientes:

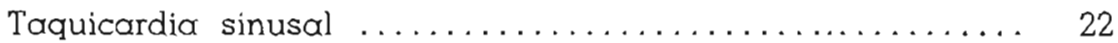

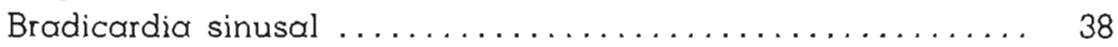

Arritmia sinusal ............................ 20

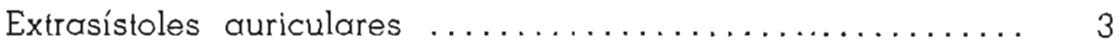

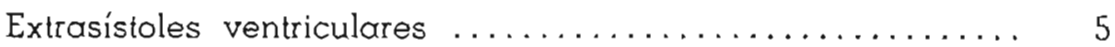

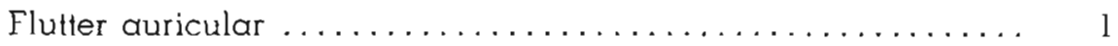

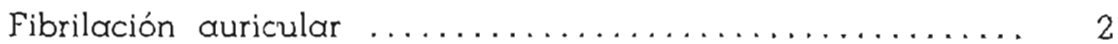

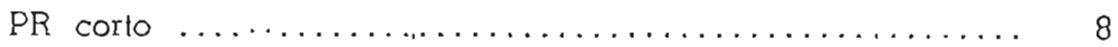

PR en límile superior normal $\ldots \ldots \ldots \ldots \ldots \ldots \ldots \ldots . \ldots . \ldots . \ldots$

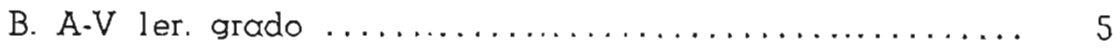

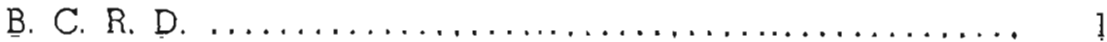


B. I. R. D. . . . . . .

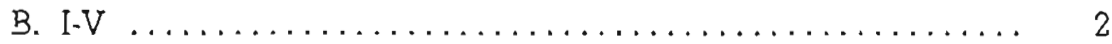

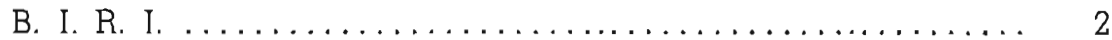

Ligero retardo de conducción de rama derecha .......... 28

QRS en límite superior normal .................... Il

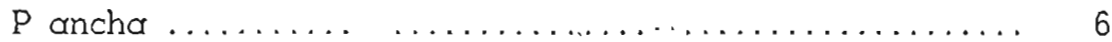

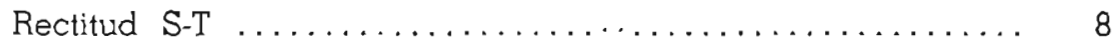

Eje eléctrico a la derecha ........................ 2

Eje eléctrico a la izquierda $\ldots \ldots \ldots \ldots \ldots \ldots \ldots \ldots \ldots \ldots, 4$

Activación auricular anormal ................... 1

Isquemia subepicárdica óntero septal $\ldots \ldots \ldots \ldots \ldots \ldots \ldots$ I

Isquemia subendocárdica póstero y ántero lateral $\ldots \ldots \ldots \ldots$ l

Isquemia inferior $\ldots \ldots \ldots \ldots \ldots \ldots \ldots \ldots \ldots \ldots \ldots \ldots . \ldots \ldots$

Cambios inespecíficos de la repolarización ventricular ..... 24

Alteración de la repolarización con daño miocárdico ........ 2

Alteración miocárdica ventricular izquierda $\ldots \ldots \ldots \ldots \ldots$ l

Hipertrofia ventricular derecha $\ldots \ldots \ldots \ldots \ldots \ldots \ldots \ldots \ldots, 2$

Hipertrofia ventricular izquierda $\ldots \ldots \ldots \ldots \ldots \ldots \ldots \ldots, 5$

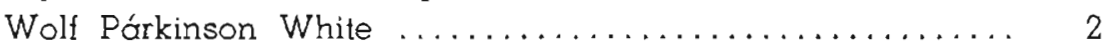

Los 362 restantes tenían valores electrocardiográficos dentro de límites normales.

La distribución del total de electrocardiogramas según grupos de edad es como sigue:

CUADRO No 4

ELECTROCARDIOGRAMAS EN 512 RESIDENTES DEL VALLE DE MOQUEGUA SEGUN GRUPOS DE EDAD

\begin{tabular}{lccc}
\hline Grupos de edad & $\begin{array}{c}\text { E. C. G. } \\
\text { efectuados }\end{array}$ & $\begin{array}{c}\text { E. C. G. } \\
\text { normales }\end{array}$ & $\begin{array}{c}\text { E. C. G. } \\
\text { anormales }\end{array}$ \\
\hline Menores de 9 años & 63 & 53 & 10 \\
$10-19$ & 134 & 107 & 27 \\
$20-39$ & 145 & 103 & 42 \\
$40-59$ & 129 & 74 & 55 \\
60 y más & 41 & 25 & 16 \\
\hline Total & 512 & 362 & 150 \\
\hline
\end{tabular}

En los 73 casos de enfermedad de Chagas diagnosticados en Moquegua, se comprobó las siguientes ạtteraciones clínịicaș: 


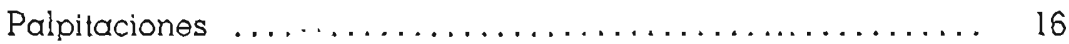

Disnea ................................... 20

Pérdida del conocimiento ....................... 2

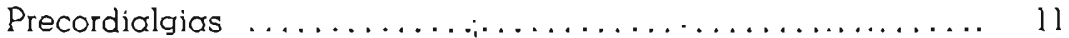

Edema maleolar ............................... 7

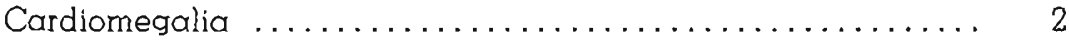

Segundo ruido aumentado de intensidad en foco pulmonar .. 2

Soplo sistólico en punta grado II/VI ................. 1

Soplo sistólico supraesternal grado II - III/VI ........... 3

Soplo diastólico aórtico grado III/VI ................ 1

De los 73 electrocardiogramas de este grupo, sólo 20 presentaron las ancrmalidades, que figuran en la relación adjunta:

Extrasístoles auriculares $\ldots \ldots \ldots \ldots \ldots \ldots \ldots \ldots \ldots \ldots \ldots$

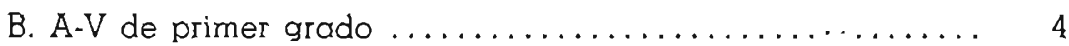

B. I. R. D. ................................ I

Ligero retardo de conducción de rama derecha ........... 4

P. ancha .................................. 3

QRS en límite superior normal $\ldots \ldots \ldots \ldots \ldots \ldots \ldots \ldots \ldots$ I

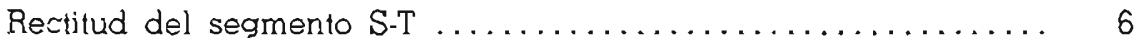

Cambios inespecíficos de la repolarización ventricular ..... 5

Alteraciones de la repolarización con daño miocárdico ántero

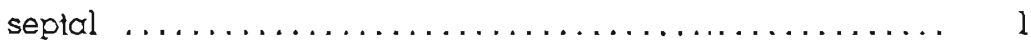

Hipertrofia ventricular izquierda $\ldots \ldots \ldots \ldots \ldots \ldots \ldots \ldots, 1$

No presentaron síntomas, signos ni alteraciones E. C. G. ..... 29

No presentaron síntomas, signos y sí alteraciones E. C. G. . 7

Presentaron síntomas, signos y alteraciones E. C. G. ........ 13

Presentaron síntomas, signos y no alteraciones E. C. G. ..... 24

CUADRO N: 5

ELECTROCARDIOGRAMAS ANORMALES EN CASOS DE ENFERMEDAD DE CHAGAS EN MAJES Y EN MOQUEGUA SEGUN GRUPOS POR EDAD

\begin{tabular}{lccc}
\hline \hline Grupos de edad & Majes & Moquegua & Total \\
\hline Menos de 9 años & 0 & 0 & 0 \\
$10-19$ & 2 & 2 & 4 \\
$20-39$ & 0 & 3 & 3 \\
$40-59$ & 0 & 9 & 9 \\
60 y más & 0 & 6 & 6 \\
\hline Total & 2 & 20 & 22 \\
\hline
\end{tabular}




\section{CUADRO N: 6}

RESUMEN DE LOS HALLAZGOS CLINICOS Y ELECTROCADIOGRAFICOS EN 105 PACIENTES CON ENFERMEDAD DE CHAGAS DIAGNOSTICADOS ENTRE 259 RESIDENTES DE MAJES Y 512 DE MOQUEGUA

\begin{tabular}{lccccc}
\hline Lugar & Positivos & $\begin{array}{c}\text { Clínicamente } \\
\text { normal }\end{array}$ & $\begin{array}{c}\text { E.C.G. } \\
\text { normal }\end{array}$ & $\begin{array}{c}\text { Clinicamente } \\
\text { anormal }\end{array}$ & $\begin{array}{r}\text { E. C. G. } \\
\text { anormal }\end{array}$ \\
\hline Majes & 32 & 22 & 13 & 10 & 2 \\
Moquegua & 73 & 36 & 63 & 37 & 20 \\
Total & 105 & 58 & 76 & 47 & 22 \\
\hline
\end{tabular}

En el grupo de Majes sólo se estudió los E. C. G. de 15 casos positivos.

\section{CUADRO N: 7}

ELECTROCARDIOGRAMAS DE LOS SUJETOS CHAGASICOS Y NO CHAGASICOS DE LOS VALLES DE MAJES Y MOQUEGUA

\begin{tabular}{|c|c|c|c|c|c|c|c|}
\hline Lugar & \multicolumn{2}{|c|}{$\begin{array}{c}\text { E. C. G. } \\
\text { efectuados }\end{array}$} & Total & \multicolumn{2}{|c|}{$\begin{array}{c}\text { E. C. G. } \\
\text { normales }\end{array}$} & $\begin{array}{l}\text { E. C. G. } \\
\text { anormales }\end{array}$ & $\%$ \\
\hline \multirow[b]{2}{*}{ Majes } & \multirow{3}{*}{243} & chagásicos & 15 & 13 & 86.67 & 2 & 13.33 \\
\hline & & no chagásicos & 228 & 193 & 84.65 & 35 & 15.35 \\
\hline \multirow{2}{*}{ Moquegua } & & chagásicos & 73 & 53 & 72.30 & 20 & 27.70 \\
\hline & 512 & no chagásicos & 439 & 309 & 70.39 & 130 & 29.61 \\
\hline Total & 755 & & 755 & $\ldots$ & $\begin{array}{r}73.91 \\
-\quad \therefore\end{array}$ & 187 & 26.09 \\
\hline
\end{tabular}

Conociendo los marcados hábitos domiciliarios del T. infestans, único transmisor de la Trypanosomiasis Americana en la región sud-occidental del Perú, decidimos investigar los efectos residuales del GAMMA BHC CERECLOR sobre esta especie, aplicándolo en un lugar de la zona estudiada del valle de Moquegua. 


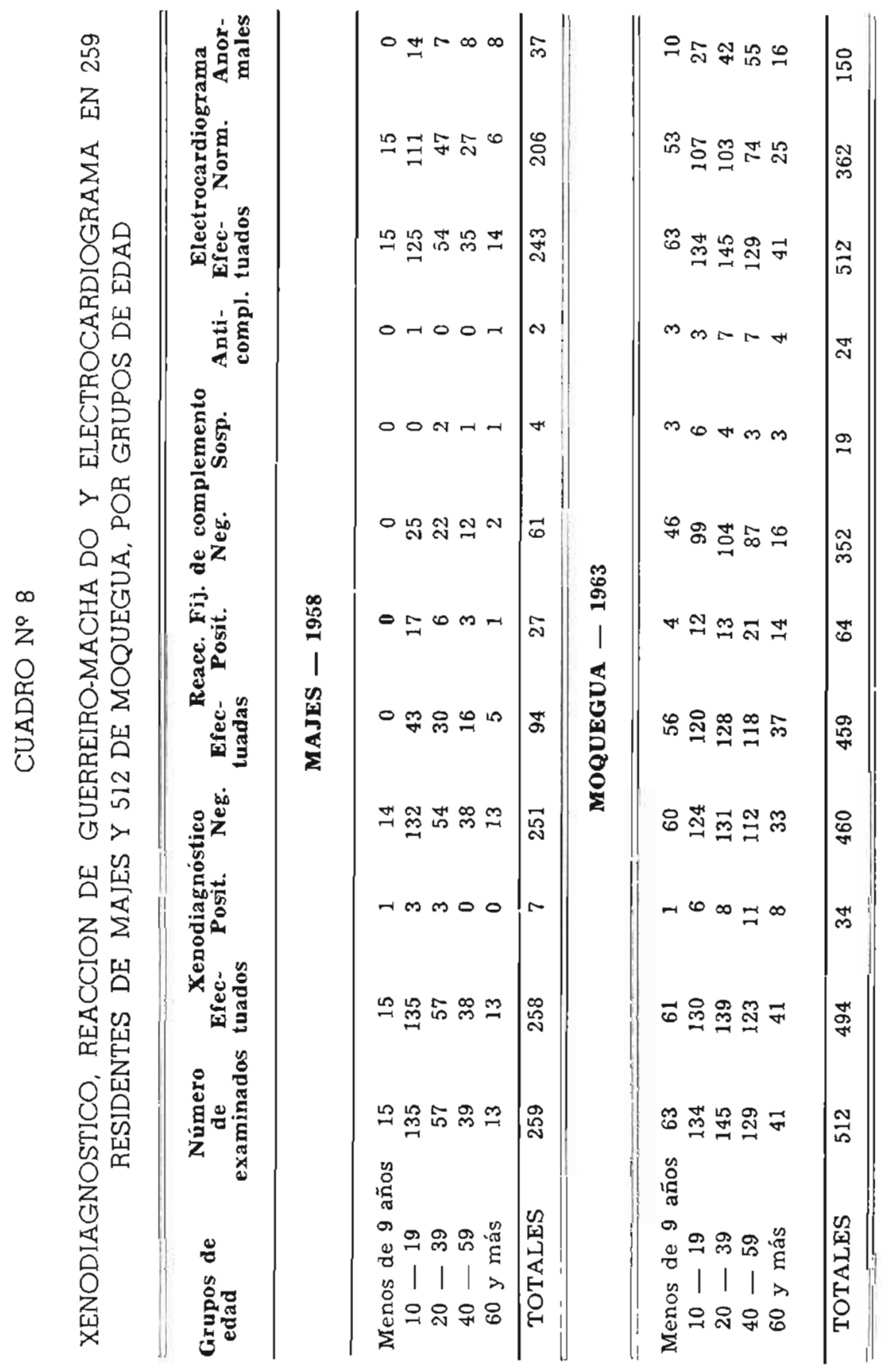


El Servicio de Erradicación de la Malaria del Perú, se encargó de rociar el referido insecticida en la totalidad de viviendas del caserío re Samegua, lugar incluído en el presente estudio. La entomóloga Srta. Carmen Villanueva, en colaboración con nosotros, dirigimos la aplicación durante la primera quincena del mes de Marzo de 1963, obteniéndose una concentración de 2 gr. por metro cuadrado y aplicándose el rociado en todo el interior de las viviendas así como en los corrales, gallineros, chiqueros y cuyeros. Los resultados preliminares obtenidos durante las primeras 72 horas posteriores a la aplicación y en el control entomológico del rociado, practicada durante los 10 meses siguientes y a intervalos de 15 a 20 días, ha permitido comprobar que no se han producido manifestaciones tóxicas sobre el personal encargado de la aplicación ni sobre los residentes de las viviendas rociadors, así como tampoco sobre los animales domésticos. La efectividad de este insecticida sobre el T. infestans, en Samegua, es alentadora, pues se comprobó la muerte de gran número de larvas, ninfas y adultos durante los primeros controles entomológicos; posteriormente, dichos controles demostraron acentuada disminución de triatóminos en el interior de las viviendas. La acción residual ha permanecido con alto grado de efectividad dirante los primeros seis meses y los residentes de las viviendas rociadas están de acuerdo en expresar que los triatóminos casi han desaparecido. Los controles realizados a partir del séptimo mes revelan que en dos o tres viviendas había chirimachas vivas.

En las visitas domiciliarias para efectuar el control entomológico del rociado, fue posible colectar triatomas en diversos estadíos evolutivos, muertos o moribundos, 6 a 10 meses después del rociado, lo que se explicaría considerando que se trataría de triatóminos que recientemente se habían puesto en contacto con las superficies rociadas.

Estos resultados preliminares en Samegua nos estimularon a extender la zona de experimentación del GAMMA BHC CERECLOR a 800,000 metros cuadrados de interiores de viviendas y durante el mes de Agosto último, se han rociado 1,120 viviendas en la ciudad de Mociuegua. Los resultados obtenidos durante los primeros cinco meses siguientes a esta nueva y más extensa zona de aplicación, son tan buenos como los observados en Samegua. Esperamos continuar efectuando controles entomológicos durante dos años en las zonas rociadas del valle de Moquegua, a fin de poder llegar a conocer la máxima duración de la efectividad de la acción residual. 


\section{COMENTARIO}

Los hallazgos efectuados en Majes duronte el año 1958 demostraron que las condiciones epidemiológicas en relación con la enfermedad de Chagas continuaban siendo tan favorables como las encontradas por nosotros en los años 1950 y 1951 (14) (15) (16); es decir, que no habion variado las condiciones favorables de la vivienda para albergar triatóminos, ya que en el $100 \%$ de las casas visitadas se comprobó la presencia de $T$. intestans y el $80 \%$ de ellas tenían triatomas infectacios. El $51.02 \%$ de T. infestans capturados en esta zona tenían trypanosomas.

En Moquegua, al igual que en Majes también se encontró que la vivienda tenía condiciones muy favorables yá que se capturó $T$. infestans en el $93.45 \%$ de las casas consideradas en la encuesta.

El $17.32 \%$ de los triatóminos capturados en este luqar estaban infectados por $T$. cruzi y el $53.13 \%$ de las viviendas teníom triatomas infeciados.

Estas condiciones eran semejantes a las encontradas en Moquegua per otros autores (17), (18), en los años 1951 y 1955.

Tanto en Majes como en Moquegua continúan crióndose animales domésticos en el interior de las viviendas y entre ellos predominan cuyes, perros y gutos de rol tan importante como reservorio de $T$. cruzi, pues de los 320 xenodiagnósticos practicados en igual número de animales domésticos en Moquegua, resultaron positivos para este trypanoscma, 12 perros, 11 cuyes, 2 cchejos y 2 gatos. Ver Cuadro $N^{\circ}$ I.

Con condiciones epidemiológicas tan farvorables, fue relativamente fácil confirmar el diagnóstico de esta enfermedad en 105 sujetos de un total de 771 examinados, entre los que se practicó 752 xenodiagnósticos y 553 reacciones de Guerrelro-Machado. Estos casos son informados por primera vez, incrementan de manera notable la casuística peruana, ya que ninguno de ellos había sido sometido a pruebas diagnósticas en anteriores oportunidades. Ninguno de los pacientes diagnosticados ahora, se encontraba en fase aguda de la enfermedad.

Una de las finalidades más importontes de esta investigación, ha sido tratar de obtener información sobre la frecuencia con que se observa síntomas, signos clínicos y alteraciones electrocardiográficas, atribuíbles a cardiopatía chagásica en una zona donde la enfermedad es endémica y no destacar las peculiaridades de la miocarditis chagásica en nuestro medio. Además, hemos creído de sumo interés hacer 
una comparación entre los hallazgos clínicos y electrocardiográficos del grupo de pacientes con enfermedad de Chagas y el grupo de sujetos residentes en esas zonas que no tenían esta trypanosomiasis.

Es interesante destacar que en menos del $50 \%$ de los 105 casos diagnosticados en Majes y Moquegua, se encontró síntomas y signos atribuíbles a compromiso cardíaco y de éstos, solamente en 22 se confirmó alteraciones electrocardiográficas. Las alteraciones de este último grupo constan en el siguiente cuadro que resulta muy interesante:

1.- Defecto de conducción derecha, sin signos electrocardiográficos definidos de bloqueo incompleto, llámese conducción derecha retardada (presencia de onda $\mathrm{S}$ empastada en DIII, V-5, V-6 y/o onda S mellada en V-l y RSr).

2.- Esbozo de conducción A-V retardada (PR $0.20^{\prime \prime}$ ).

3.--Rectitud del segmento ST en derivaciones izquierdas.

4. - Onda $P$ ancha.

5.- B. A-V de primer grado.

6.- Asociación de $\mathrm{P}$ ancha, rectitud del segmento ST y extrasístoles auriculares.

7.- Asociación de P ancha, B. A-V de primer grado, rectitud del segmento ST e hipertrofia ventricular izquierda.

8.- No se encontró casos de B. C. R. D.

Es así mismo importante anotar que ninguno de los casos positivos presentó signos clínicos de insuficiencia coronaria ni enfermedad valvu. lar y que el orden de alteraciones electrocardiográficas arriba indicado, guarda secuencia con la edad del sujeto y su tiempo de permanencia en la zona endémica, es decir, que mientras mayor fue la edad y tiempo de residencia, más evidentes fueron las alteraciones electrocardiogróficas.

El mayor número de electrocordiogramas con alteraciones, se observó en el grupo de 40 a 59 años de edad, siguiendo en frecuencia el de 60 y más y los grupos de 10 a 19 y 20 a 39 años.

Al comparar los hallazgos electrocardiográficos en sujetos chagásicos y no chagásicos residentes en la misma zona endémica, encontramos que, en Majes, de 15 chagásicos hubo dos con anormalidades y entre 228 no chagásicos 35 presentaron alteraciones electrocardiográficas. Comparación similar se realizó en Moquegua comprobándose que 20 de los 73 chagásicos, tenían alteractones electrocardiográficas mientras que 130 las presentaron en el grupo de los no chagásicos (309). 
Hemos encontrado 6 sujetos con B. C. R. D.; 8 con B. I. R. D. y 14 con B. A-V de primer grado, en el grupo no chagásico, mientras que en el que tenía Trypanosomiasis Americana, sólo se comprobó 0,1 y 6 de las mencionadas alteraciones, respectivamente. En el primer grupo hubo 24 con ligero retardo de conducción de rama derecha mientras que sólo en 4 del segundo grupo, se observó esta alteración. Las alteraciones de la onda $P$ estuvieron presentes 6 veces en el primer grupo y sólo 3 en el segundo; en 11 de los que no tenían enfermedad de Chagas se encontró el complejo QRS en límite superior normal, carácter que sólo estuvo presente en uno del grupo chagásico; así mismo en los primeros hubo 24 personas con cambios inespecíficos de la repolarización ventricular, alteración que sólo estuvo presente en 5 del grupo chagásico. Hipertrofia ventricular izquierda se encontró en 5 personas que no tenían enfermedad de Chagas y en dos de las que si tenian esta enfermedad.

Extrasistolia ventricular, únicamente se encontró en los sujetos no chagásicos, lo mismo que flutter auricular y fibrilación auricular. Las desviaciones del eje eléctrico, tomto a la derecha como a la izquierda, sólo fueron halladas en las personas que no tenian enfermedad de Chogas.

Estos hallazgos demostraron claramente que no sólo las alteraciones electrocardlográficas fueron más frecuentes y variadas en el grupo no chagásico sino también, que no nos fue posible encontrar, con la frecuencia que describen muchos autores, alteraciones electrocardiográficas propias de la miocarditis chagásica. Esto nos indicaría que en el Perú no es frecuente la miocarditis chagásica y que los signos electrocardiográficos de los sujetos que la tienen, son bastante discretos y no se presenta con los caracteres alarmantes que han sido señalados en otros países.

No es posible hacer muchos comentarios en relación con la efectividad de la acción residual del GAMMA BHC CERECLOR, usado a la concentración de $2 \mathrm{gms}$. por metro cuadrado, debido a que há transcurrido 10 meses desde su primera aplicación en Samegua y cinco meses desde que fue aplicado en la ciudad de Moquegua, insecticida que po: primera vez se usa en el Perú; pero si queremos destacar que en este lapso ha demostrado ser bastonte eficaz contra el T. infestans, marteniendo su acción residual en el mismo nivel de efectividad durante los seis primeros meses, efectividad que comienza a decrecer lentamente entre el $7^{\circ}$ y $10^{\circ}$ mes. 
También debemos señalar que durante los 10 meses posteriores a su primera aplicación, no se han presentado manifestaciones tóxicas sobre las personas y animales domésticos de las vlviendas rociadas.

\section{SUMARIO}

Se ha efectuado amplio estudio epidemiológico, clínico, electrocardiográfico y profiláctico en relación con la entermedad de Chagas en los centros poblados más importantes de dos valles del Sur del Perú, demostrándose la presencia de factores epidemiológicos muy favorables para la presencia de esta enfermedad.

Casi el $100 \%$ de las viviendas tenían $T$. infestans y en más del $50 \%$ de ellás, estos triatomas estaban infectados por $T$. cruzi.

En el interior de las casas se crían numerosos y variados animales domésticos entre los que predominan cuyes, conejos, gatos y perros, que tienen rol importante como reservorio de $T$. cruzi.

Se ha practicado 320 xenodiagnósticos a igual número de animales de las viviendas de Moquegua, resultando 12 positivos entre 113 perros, 11 positivos en 62 cuyes, 2 positivos entre 68 gatos y 2 positivos en $3 C$ coriejos. Los restantes ( 27 cerdos, 8 caballos, 6 burros y 6 cabras) dieron resultado negativo.

Hemos practicado 752 xenodiagnósticos y 553 reacciones de Guerreiro-Machado en un total de 771 personas residentes de esos valles que no fueron previamente seleccionados, lo que permitió el diagnóstico de 105 casos nuevos. Se ha comprobado que la sintomatología y alteraciones electrocardiográficas en estos casos nuevos son muy discretas; es más, esta pobre sintomatología y signos electrocardiográficos fueron, paradógicamente, más frecuentes en los no chagásicos que en los chagásicos.

El compromiso cardíaco en los 105 casos referidos no ha sido frecuente ni ha tenido los caracteres de severidad que han sido descritos en otros países de América.

Se ha investigado la efectivtdad del Gamma-B.H.C.-Cereclor contra el T. intestans en los centros poblados del valle de Moquegua, obtenléndose resultados bastante alentadores diez meses después de su aplicación.

\section{SUMMARY}

1. A wide scale epidemtologic, clinical, electrocardiographic and prophylactic study has been corrled out in residents relating to Chagas' Disease in Majes and Moquegua valleys of Southern Perú. This study 
has shown the presence of very favorable epidemiologic conditions for the appearance of this disease.

2. One hundred per cent of the dwelling had T. infestans and $T$. cruzi was found in over $50 \%$ of them.

3. Seven hundred and fifty five electrocardiograms, 752 xenodiagnostic tests and 553 Machado-Guerreiro complement fixation tests were periomed in 771 non-selected residents of these areas and 105 new cases of the disease could thus be diagnosed.

4. All of them had a very mild and atypical clinical picture and the electrocardiographic findings were minimal unlike those describe by others authors elsewhere. On the other hand, the more striking clinical and E. C. G. findings were found in the non-chagasic population.

5. A coment has been made about the important epidemiologic meaning of household animals as reservoirs of $T$. cruzi. Considereing this fact 320 xenodiagnostic tests were performed. Twelve out of 113 dogs examined were found to be positive, as well as 11 out of 62 guinea pigs, 2 out of 65 cats and 2 out of 32 rabbits. The others animals studied gave negative results.

6. The effectiveness of Gamma-BHC-Cereclor against $T$. infestans, was studied in two towns of Moquegua Valley and has given promising results, to date, 10 months after its used.

\section{AGRADECIMIENTOS}

Expresamos nuestro agradecimiento al señor Decano de la Facultad de Medicina de San Fernando, Dr. Alberto Guzmán Barrón, por habernos brindadc toda clase de facilidades para efectuar las investigaciones durante el año 1963; al Dr. Aiberto Hurtado, ex-Decano de la Facultad, al Dr. Rafael Alzamora F., y al Dr. Marcos Roittman por la ayuda y colaboración que nos prestaron durante las investigaciones realizadas en 1958. Al Jefe del Departamento de Medicina. Dr. Carlos Lanfranco; al Dr. Hugo Pesce Profesor Principal de Medicina Tropical, al Dr. Luis D. Gutiérrez ex-Profesor de la misma; a los médicos cardiólogos y al técnico electrocardiografista del Departamento de Medicina les expresamos nuestro agradecimiento.

Agradecemos en forma especial al Sr. ex-Ministro de Salud Pública Dr. Victor Solano Castro, al Mr. Ministro actual, Dr. Javier Arias Stella, al Sr. Director General de Salud Pública, Dr. Carlos Quiroz S., al personal de la Campaña de Erradicación de la Malaria, en especial a su Director Dr. Enrique Villalobos y a la Srta. Entomóloga Carmen Villanueva. A los médicos del Area de Salud de Tacna y de la Unidad de Salud de Moquegua. A las autoridades politicas de Moquegua y al Presidente de la Junta de Obras Públicas.

Nuestra gratitud al Dr. Amador Neghme y a la Dra. Knierim, del Dpto. de Farasitología de la Universidad de Chile, a los médicos del Hospital de Beneficencia de Moquegua y al médico Sanitario de Aplao por su magnífica cọlaboración. 


\section{B I B L I O G A F I A}

1. Escomel, Edmundo., 1919. La trypanosomias humaine existe dan les forts orientalis du Perou. Bull. Soc. Pat. Exot., París, 12: 723-726.

2. Escomel, Edmundo., 1917. Insectos hemípteros, heterópteros, hematófagos en nuestros valles del sur del Perú. Encierran formas protozoaicas herpetómonicas. Existe entre nosotros la enfermedad de Chagas? La Ref. Méd., Lima, 3: 121-122.

3. Cornejo-Donayre, A., 1958. Enfermedad de Chagas. Estado actual en el Perú. Pro, 6th Intern'l Congr. Trop. Med. \& Malaria, Lisboa. Imprensa Portuguesa, 3: 298-324.

4. Rosenbaun, M. B. y Gerisola, J. A., 1958. Consideraciones épidemiológicas sobre la enfermedad de Chagas en la Argentina, Rev. Conf. Méd. Panamericana, 5: 197-201.

5. Rodriguez-Rivas, J., 1961. Enfermedad de Chagas y Cardiopatía Chagásica en Bolivia. Pub. Fac. Med. Univ. Mayor de San Simón, Cochabamba.

6. Laranja, F. S., Dias, E., Nobrega, G. y Miranda, A., 1956. Chagas'Disease. A Clinical Epidemiologic and Pathologic Study. Circulation, 14: 1035.

7. López, J. E. y Maekelt, G. A., 1960. La Miocarditis crónica chagásica en adultos. Descripción clínica de casos puros de Miocarditis Crónica Chagásica sin asociación con otras enfermedades. Arch. Venezolanos de Med. Trop. \& Parasit. 3: 107-124.

8. Schenone, H. y Niedman, G., 1957. Nuevos aportes al estudio de la cardiopatía chagásica crónica en Chile. Bol. Chileno Parasitol., 12: 2-7.

9. Alzamora, F. M., 1958. Caso de miocarditis chagásica observado en Lima en Noviembre de 1928. Rev. Peruana de Cardiología, 7: 327.

10. Peralta, A. V., Cornejo-Donayre, A., Rodríguez, L. J., Domínguez, P. y Berrocal A., 1960. Investigación sobre la presencia de Enfermedad de Chagas en el personal de la Policía Peruana. An. Fac. Med., Li$\mathrm{ma}, 41: 428-453$.

11. Peralta, A. V., Cornejo-Donayre, A., Otero, A. G., Castañeda, L. P., Rodríguez, L. J., Domínguez, P. y Berrocal, A., Enfermedad de Chagas Familiar. Un caso de Edema Agudo del Pulmón y un Caso de Enfermedad de Chagas Posiblemente congénito. An. Fac. Med., Li$\mathrm{ma}, 44: 386-404$.

12. Peralta, Aurelio, 1958. Clínica de Miocarditis Chagásica. Revista del Viernes Médico. 9: 1-20.

13. Montesinos, J., Náquira, C. Ordóñez, R., Paredes, O., Rojas, V., Rondón, V., Sevillano, G., Tejada, A. y Vargas, J., 1960. Contribución al estudio de la enfermedad de Chagas en el Perú. Observaciones en el valle de río Sihuas, 1956. (Provincia de Arequipa, Departamento de Arequipa). An. Fac. Med., 43: 599-715.

14. Cornejo-Donayre, A., y Lumbreras, C. H., 1950. Estudios preliminares sobre epidemiologia de la enfermedad de Chagas en el Valle de Majes. Arch. Per. Patọl. Clin., Lima, 4: 121-130. 
15. Cornejo-Donayre, A. y Lumbreras, C. H., 1951. Encuesta epidemiológica sobre la enfermedad de Chagas en el valle de Majes. Presentación de diez nuevos casos. Arch. Per. Patol. Clín., Lima, 5: 79-106.

16. Cornejo-Donayre A. y Lumbreras, C. H., 1951. Estudios preliminares sobre epidemiología de la enfermedad de Chagas en el valle de $\mathrm{Ca}$ maná. Arch. Per Patol. Clín., Lima, 5: 107-112.

17. Garcia-Cáceres, U., 1951. Contribución al estudio de la patologia endémica de los valles del extremo sur de la Costa Peruana. La enfermedad de Chagas en el valle de Moquegua. Rev. Med. Exp., Lima, 8: 227-243.

18. Herrer, A., Trypanosomiasis Americana en el Perú. I. El insecto vector y los animales que actúan de reservorio de la enfermedad de Chagas en la región sudoccidental. Rev. Med. Exp., Lima, 9: 23-37.

19. Bozicevich, J., Hoyen, H. and Walston, V. M., 1946. A method of conducting the $50 \%$ of hemolysis and poind complement-fixation for parasitic diseases. Pub. Health Rep., 61: 529-534.

20. Knierim, F., 1958. Técnica de la reacción de fijación del complemento según el $50 \%$ de hemólisis de Bozicevich aplicado al diagnóstico de la enfermedad de Chagas. Bol. Chileno Parasitol., 13: 75-78.

21. Cubas, N. E., 1963. Reacción de Guerreiro-Machado en 472 residentes de Lima con residencia anterior en otros Departamentos. Preparación del antígeno y método dej $50 \%$ de Hemólisis de Bozicevich. Tesis de Bachiller en Medicina. Lima, Fac. Med. San Fernando, U. N. M. S. M. 


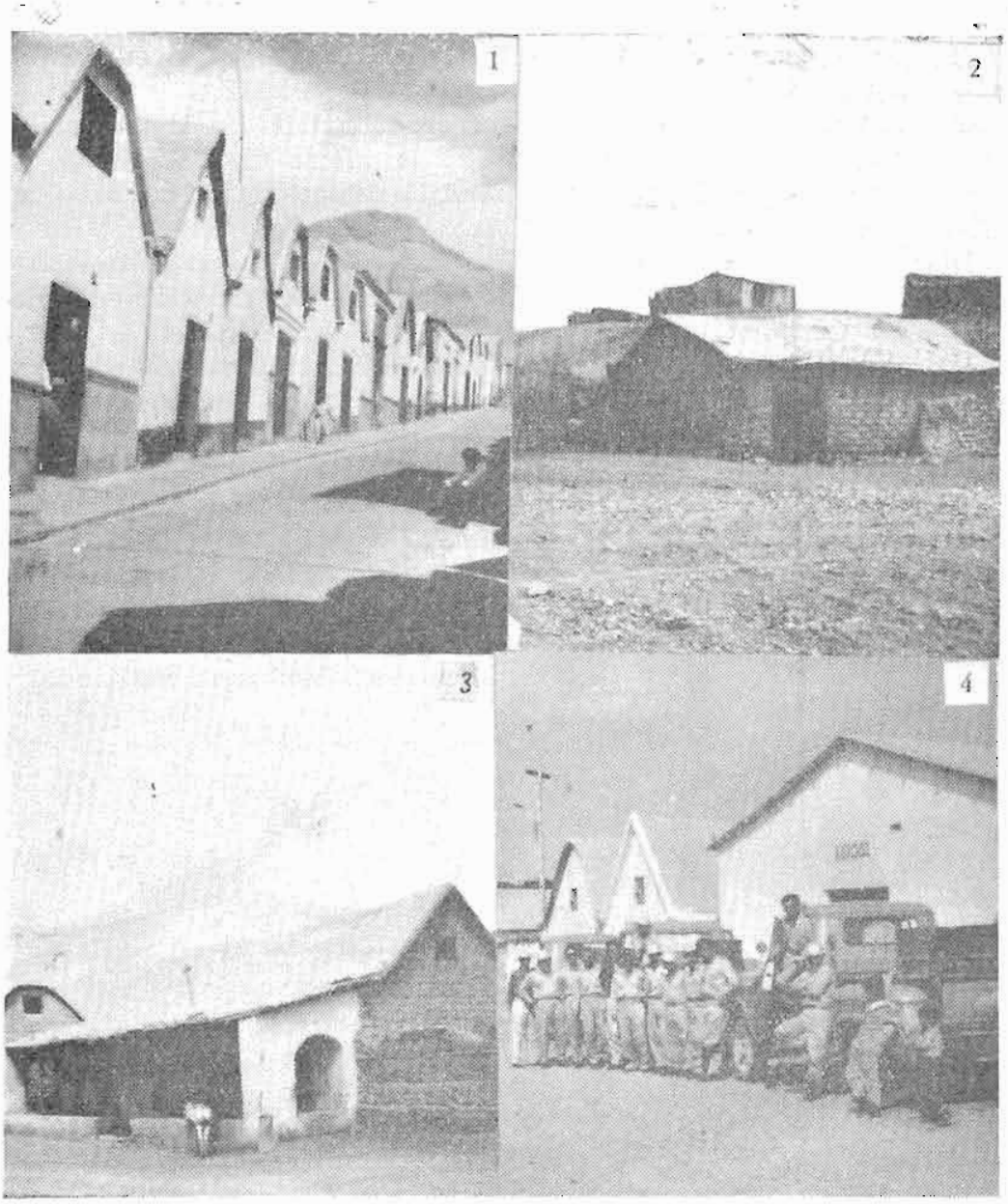

1.- Casas de Moquegua, con su techo en caballece.- 2-3.-Casas de Samegua; se aprecia sus caracteri sticas generales y material de, construcción. 4.- Brigada de rociadores del S N E M en Samegua. 


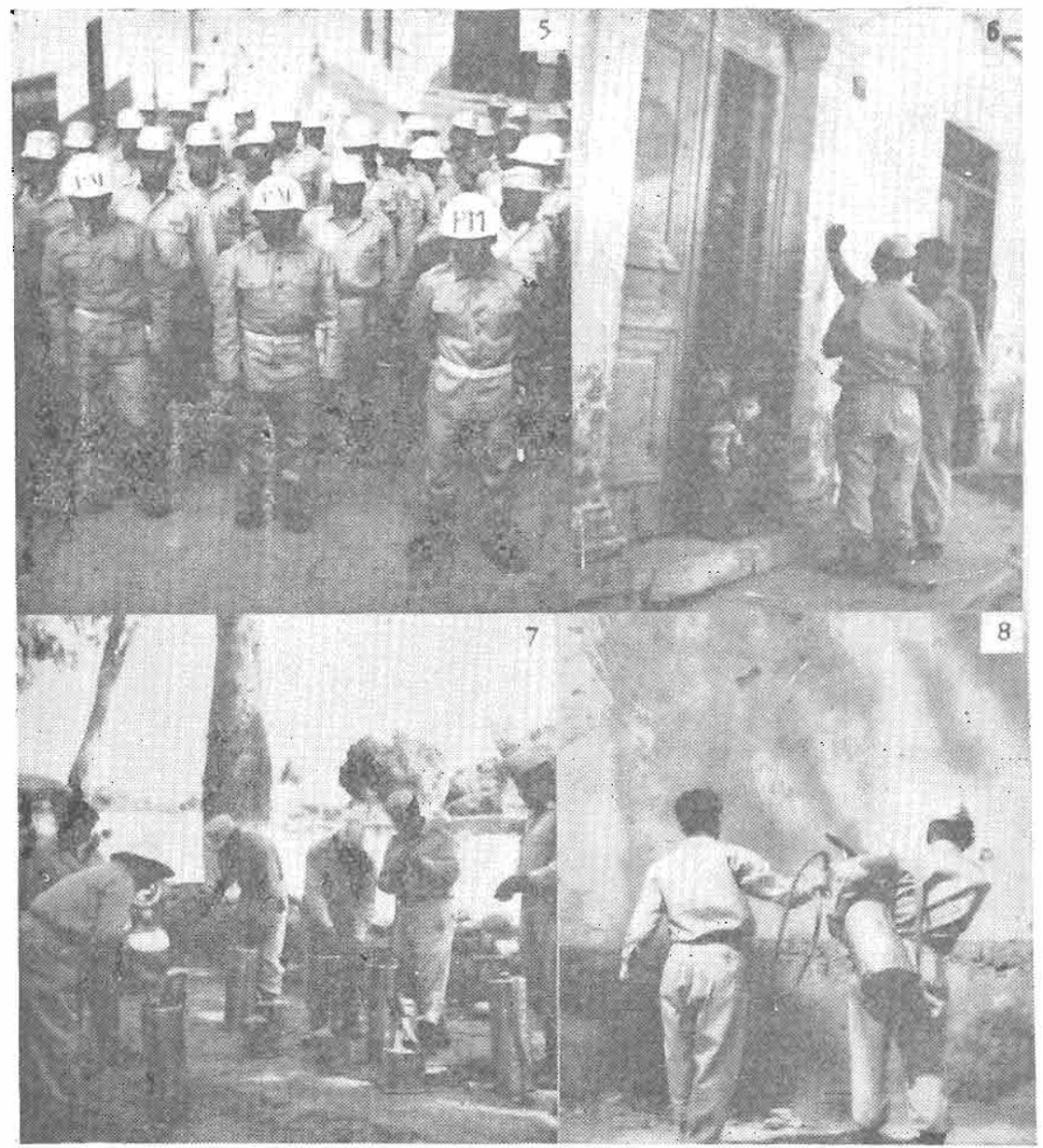

5.- Parte del personal del Batallón de Infantería $N^{\mathcal{Q}} 41$ de Infantería de Moquegua, que colaboró en el rociado. $6-7$ - 8.: Preparación y entrenamiento del personal mil itar en I a numeración de las casas rociadas, menejo del equipo y técnica de rociado. 


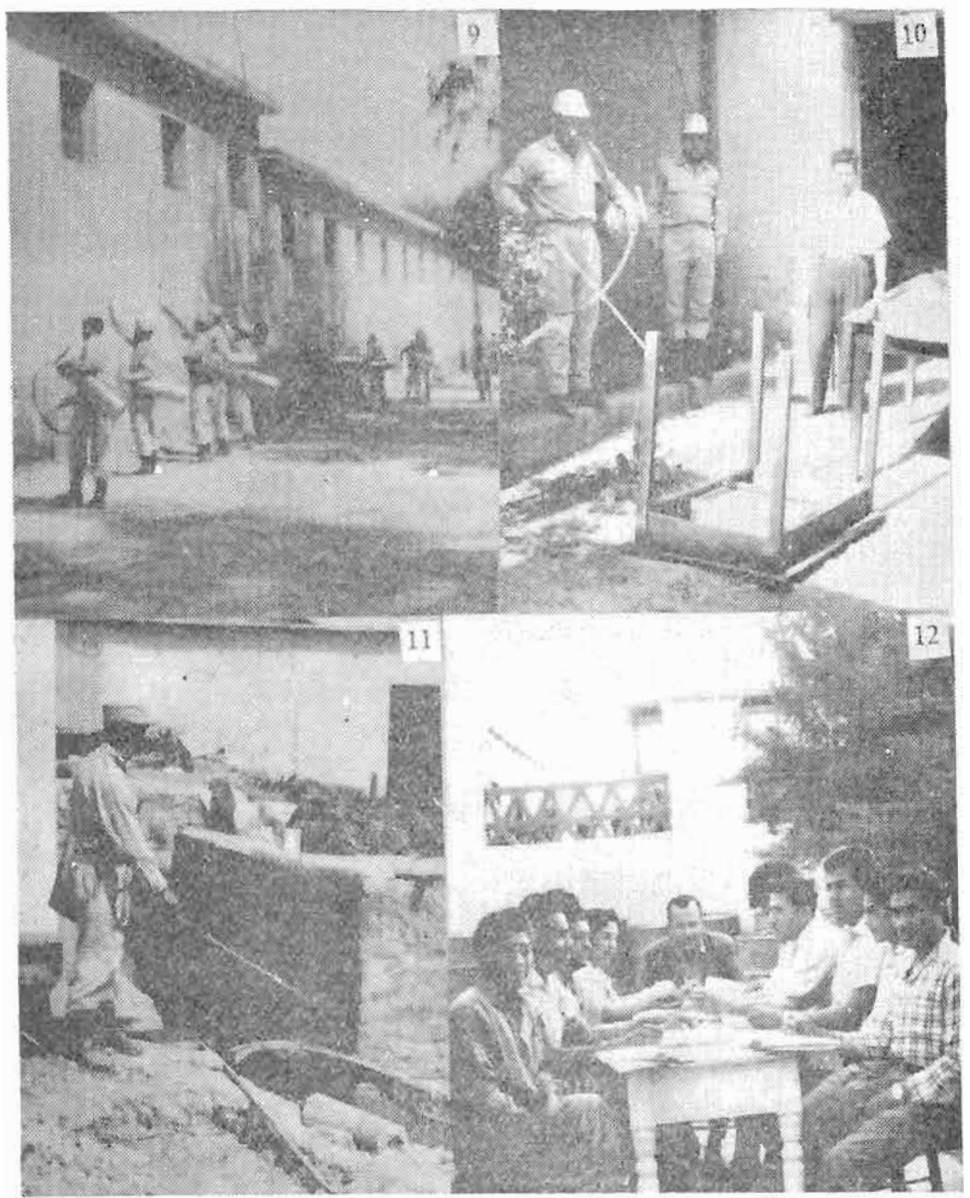

9.- Entrenamiento del personal militar para el rociado. 10.- Rociado total del interior de la vivienda. 11.- Rociado del exterior de corrales. 12.- Reunión diaria con Inspec. tores y Jefes de Brigada para comentar el trabajo realizado y programar el del dís siguienre. $\varepsilon$ 
V.C. - Ne 248 - 52 años - Nacido en Moquegua

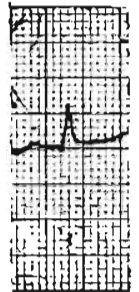

DI

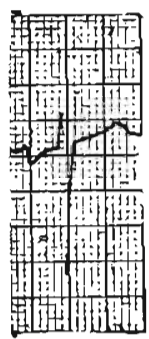

VI

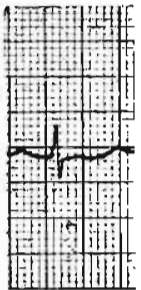

DII

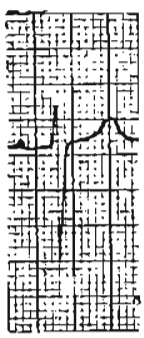

v2

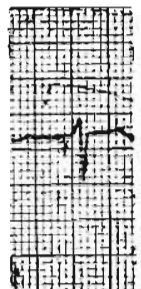

DIII

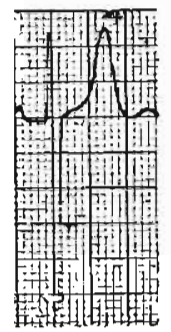

v3

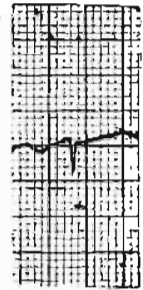

aVR

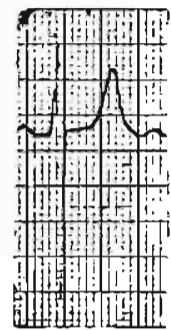

V4

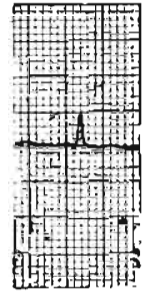

aVL y aVF

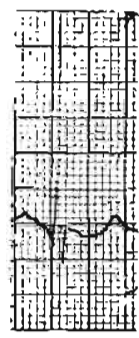

V5
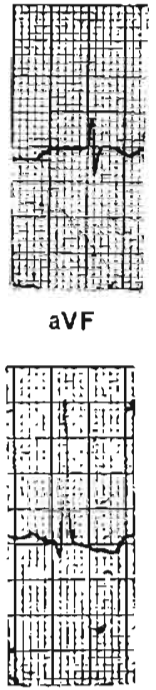

V6

Fıg. 13.-- Xencdiagnóstico positivo Nótese la conducción A.V. retordada (Bloqueo A.V. de ler. grado). La onda P es binodal. Los voltajes de las derivociones precordiales izquierdos sugieren hipertrofia ventricular izquierda

I. H. - Ne 132 - 32 años - Reside en Samegua 13 años

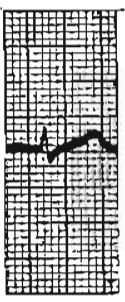

DI

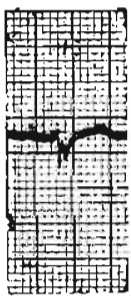

V1

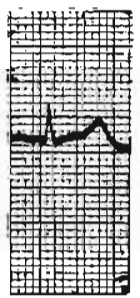

DII

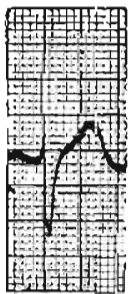

V2

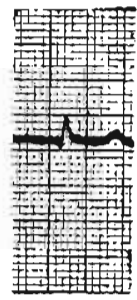

DIII

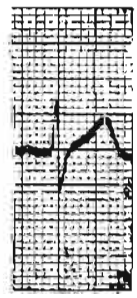

v3

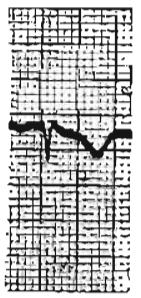

aVR

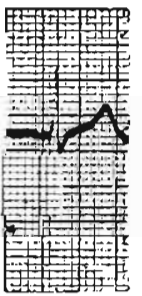

V4

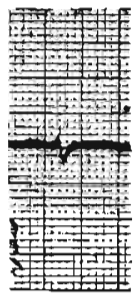

aVL

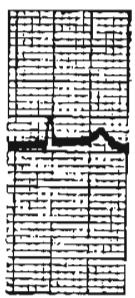

ave

Fig 14 - Sujeto con Reacción de Fijoción de complemento y xenodiagnóstico positivos. Sólo se observo un esbozo de conducción derecha retardado en VI. 
J. M. - N9 122 - 24 años - Nacida en Samegua 7 años

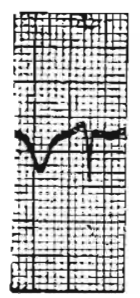

OI

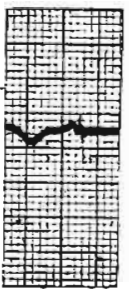

VI

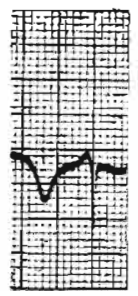

DII

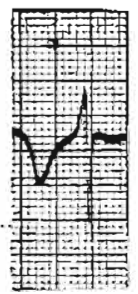

DIII

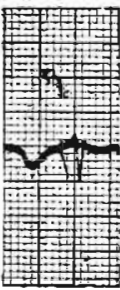

$\mathrm{V} 2$

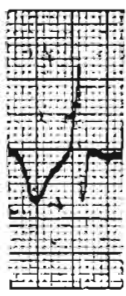

aVR

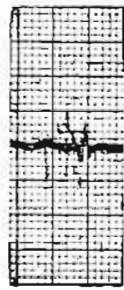

$\mathrm{V} 4$

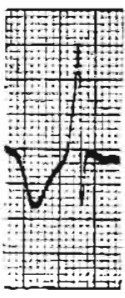

aVL

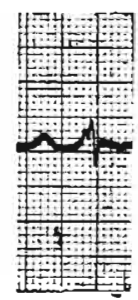

aVF

Fig. 15.- Este pociente con R.F.C. y xenodiognóstico positivos, es otro coso con defecto de conducción derecho.

J. V. - № 28 - 8 ã̃os - Nacido en Samegua

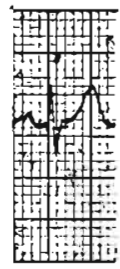

Dl

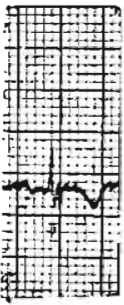

V1

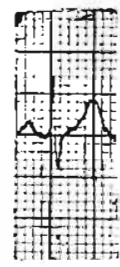

DII

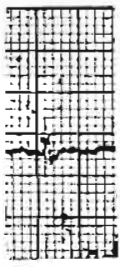

DIII

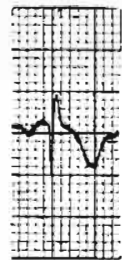

aVR

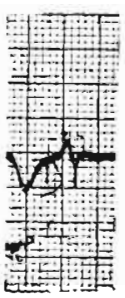

V5

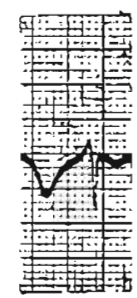

V6 
P. S. - № 23 - 44 años - Reside en Samegua 30 años

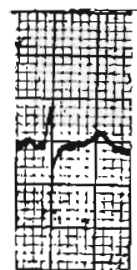

ol

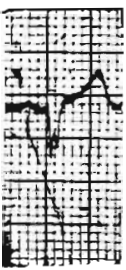

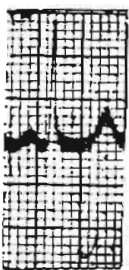

DII

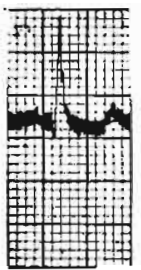

DIII

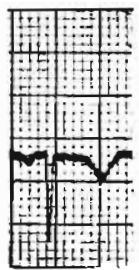

aVR

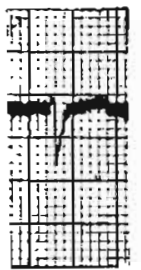

aVL

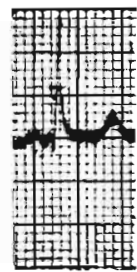

aVF

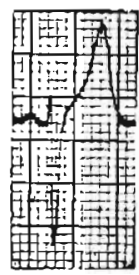

$\mathrm{V} 2$

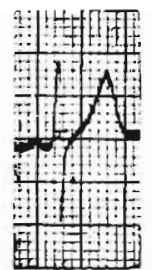

V3

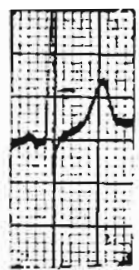

V4
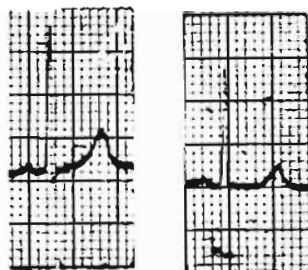

V6

Fig. 17.- Sujeto con R.F.C. positivo. Mellomiento de S en VI y rectitud segmentario de ST en DII, DIll y aVF estó presente.

D. F. - N222 - 13 años - Reside en Samegua 10 años

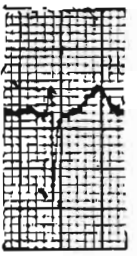

DI

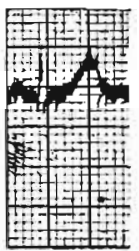

DII

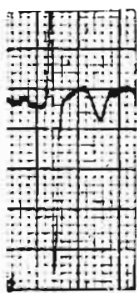

V2

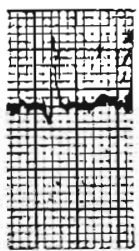

DIII

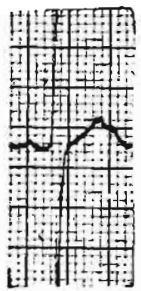

V3

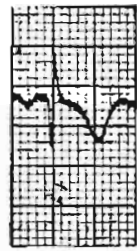

aVR

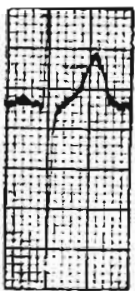

V4

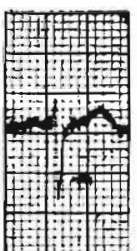

aVL
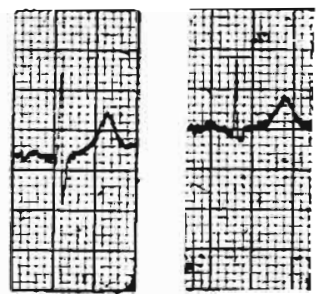

V5

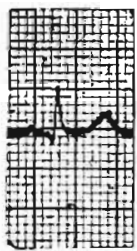

aVF

Fig. 18.- R.F.C. positiva. Clínicomente asistomático. No presenta ruidos agregados anormales a la auscultación del corazón. Eje eléctrico es derecho. El potrón de $r R^{\prime}$ en $V I$ con inversión de ondo $T$ en $V 2, V 3$ sugieren sobrecarga ventricular derecha. El tiempo de conducción es normal. 
E. V. Z. - No 80 - 51 años - Nacida en Moquegua

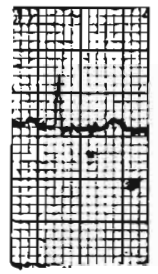

DI

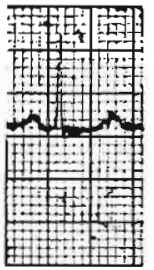

DII

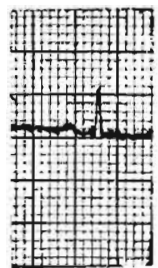

DIII

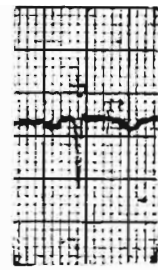

aVR

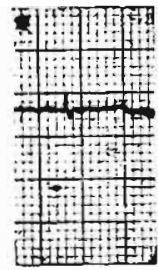

aVL

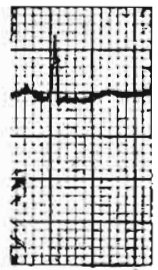

aVF

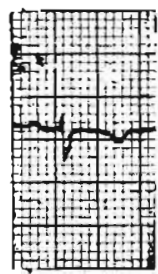

V1

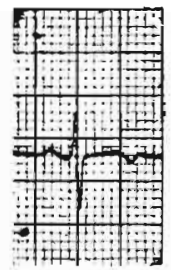

V2

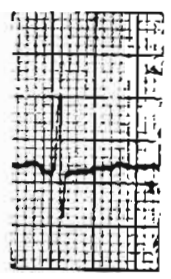

v3

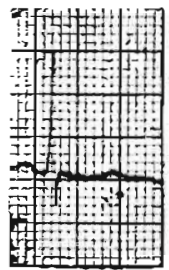

V4

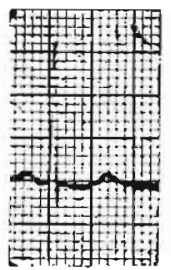

v5

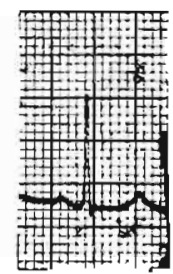

V6

Fig. 19.- R.F.C. y xenodiagnóstico positivos. Hoy cambios inespecificos de ST y T. Se oprecia rectitud del segmento ST especialmente en derivaciones Standar.

M. C. V. - № 256 - 65 años - Reside en Moquegua 42 años

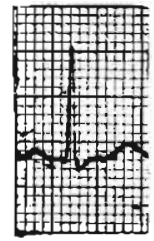

DI

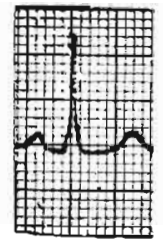

DII

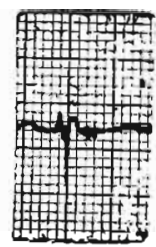

DIII

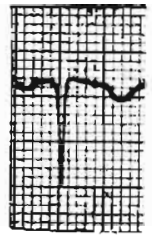

aVR

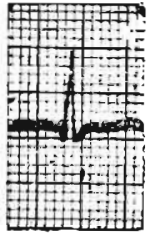

aVL

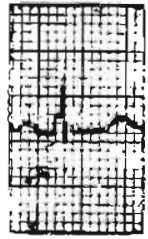

aVF
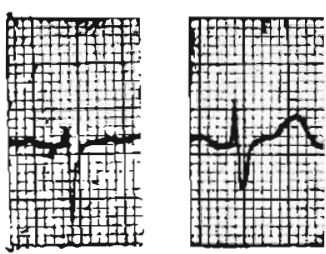
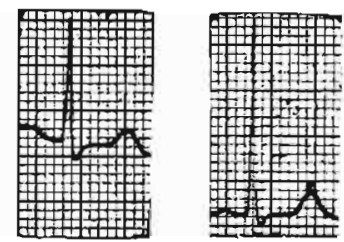

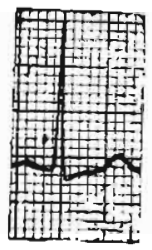

V5

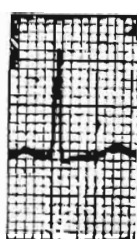

V6

V1

V2

V3

V4

Fig. 20.- Con R.F.C. y xenodiognóstico positivos. Lo rectitud seomentaria de ST
es mos notoria especialmente en DII, DIII V3, V4, V5 y V6. es mos notoria especialmente en DII, DIII V3, VA, V5 y V6. 
E. A. - $N^{2} 120 \cdot 60$ años - Nacida en Samegua

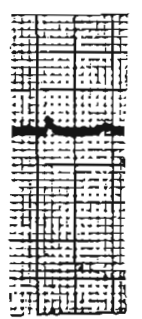

DI

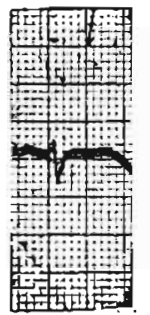

V1

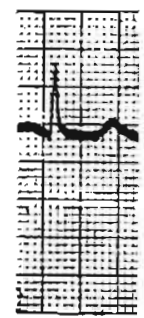

DII

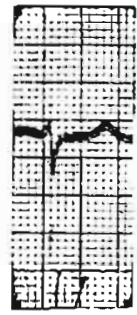

V2

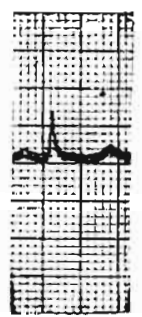

DIII

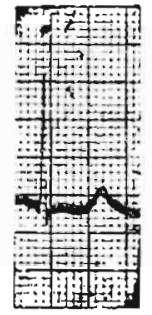

V3

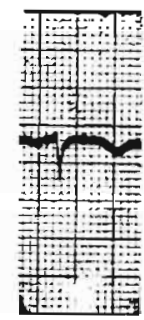

AYR

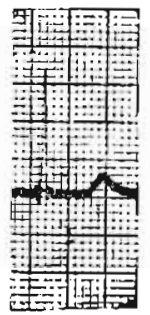

V4

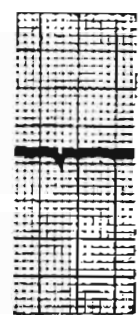

aVL

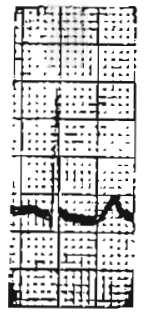

V5

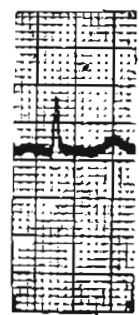

aVF

Fig. 21.- Sujeto con R.F.C. y xenodiognóstico positivos. EI E.C.G. es similor of de la fig. 20

A. P. - N 50 - 40 años - Nacida en Samegua

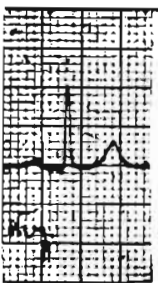

D1

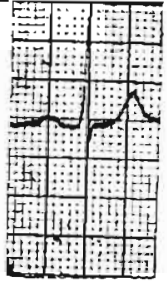

DII

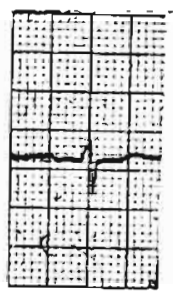

DIII

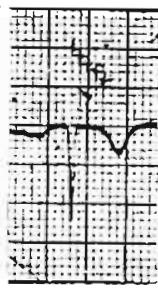

aVR

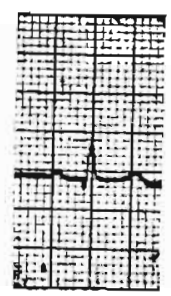

aVL

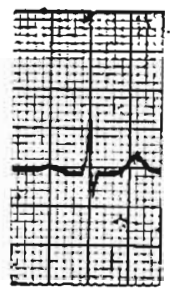

aVF

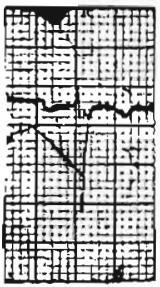

V1

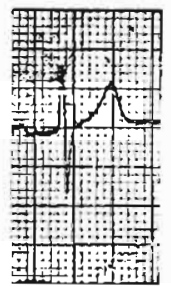

V2

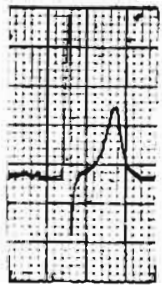

v3

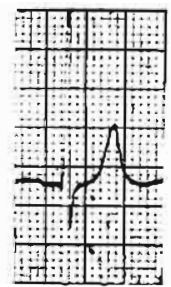

V4

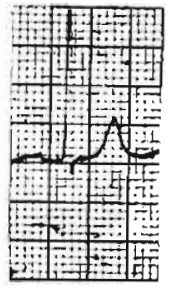

V5

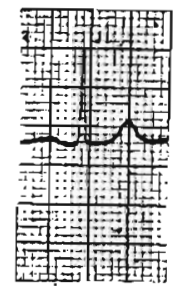

V6

Fig. 22.- R.F.C. positiva. Clinicamente se ousculta un soplo sistólico gra. do 111 en apex. Moderoda cardiomegalia. No se ousculta soplo diastólico. El trazado muestro ondas $P$ an-has en todas las derivaciones. 